\title{
ON THE REPRESENTATION THEOREM OF SCATTERING
}

\author{
BY P. MASANI ${ }^{1}$
}

Communicated by P. R. Halmos, October 20, 1967

1. Introduction. We shall show that Theorem 1.1 on the Outgoing Translational Representation of a unitary group due to Lax and Phillips [4, Theorem 1], [5, Corollary 3.2, p. 51] follows readily from the Wold Decomposition for isometric flows obtained by the writer [7] in 1962. Our treatment will also reveal the "accessory" Hilbert space, give an explicit integral representation for the isometry occurring in the conclusion of the theorem ( $W$ and $\Sigma$ in 1.1), and place in perspective the Spectral Representation due to Sinai [9], (cf. [5, Theorem 3.1, p. 50]).

1.1. TheOREM (LAX-PhilliPS). Let $\mathfrak{F}_{0}$ be an outgoing subspace of $a$ (complex) Hilbert space $\mathfrak{H C}$ relative to the strongly continuous group $\left(U_{t},-\infty<t<\infty\right)$ of unitary operators on $\mathfrak{F C}$ onto $\mathfrak{H C} ;$ i.e. let

(i) $\forall t>0, \quad U_{t}\left(\mathcal{H C}_{0}\right) \subseteq \mathcal{H}_{0}$,

$$
\text { (ii) } \bigcap_{i>0} U_{t}\left(\mathcal{F C}_{0}\right)=\{0\}, \quad \text { (iii) cls. } \bigcup_{t<0} U_{t}\left(\mathcal{F C}_{0}\right)=\mathfrak{H C} \text {. }
$$

Then there exists a Hilbert space $W$ and a unitary operator $\Sigma$ on $L_{2}((-\infty, \infty) ; W)$ onto $\mathcal{H C}$ such that ${ }^{2}$

$$
\Sigma\left\{L_{2}([0, \infty) ; W)\right\}=\mathcal{F}_{0} ; \quad \Sigma \circ \tau_{-t} \circ \Sigma^{-1}=U_{t}, \quad-\infty<t<\infty,
$$

where $\tau_{t}$ is translation through $t$, i.e. $\left(\tau_{t} x\right)(s)=x(t+s)$.

We shall first indicate our proof in the discrete case. Replace the real $t$ in 1.1 by the integer $k$ and $U_{t}$ by $U^{k}$. The hypothesis $1.1(\mathrm{i})$ then tells us that (Rstr. $\mathfrak{F e}_{0} U^{k}, k \geqq 0$ ) is a semigroup of isometries on $\mathfrak{H C}_{0}$ to $\mathfrak{H C}_{0}$. Now for any discrete semigroup $\left(V^{k}, k \geqq 0\right)$ of isometries on a Hilbert space $\mathfrak{H C}$ to $\mathfrak{H C}$ we have the Wold decomposition, (cf. Halmos $[2$, Lemma 1] or $[7,(1.1)])$ :

$$
\begin{aligned}
& \mathfrak{H C}=\mathfrak{H}_{\infty}+\sum_{k=0}^{\infty} V^{k}(W), \quad \mathfrak{F}_{\infty} \perp \sum_{k=0}^{\infty} V^{k}(W) \\
& \mathfrak{H}_{\infty}=\bigcap_{\mathrm{d}} V_{\mathfrak{k} \geq 0}^{k}(\mathfrak{H C}), \quad W \underset{\mathrm{d}}{=}\{V(\mathfrak{H C})\}^{\perp} \text {. }
\end{aligned}
$$

Hence in our case, cf. 1.1(ii),

1 This work was supported by the Office of Naval Research.

${ }^{2}$ In this paper $L_{2}([a, b] ; W)$ shall denote the set of all functions in $L_{2}((-\infty, \infty)$; $W$ ) essentially supported on $[a, b]$. Rstr.s $F$ means: restriction of $F$ to $S$. For $=\mathrm{d}$ read "equals by definition." 


$$
\mathfrak{H C}_{0}=\sum_{k=0}^{\infty} U^{k}(W), \quad W=\mathfrak{H}_{0} \cap\left\{U\left(\mathfrak{F}_{0}\right)\right\}^{\perp} .
$$

From this we easily infer as in $[5$, p. 38] that

$$
\mathfrak{H}=\operatorname{cls} . \bigcup_{\nu=-\infty}^{\infty} U^{\nu}\left(\mathcal{H C}_{0}\right)=\sum_{k=-\infty}^{\infty} U^{k}(W) .
$$

Now the last term is clearly the set of all vector-sums $\sum_{k=-\infty}^{\infty} U^{k}\{x(k)\}$, where $x(\cdot)$ is in the space $l_{2}(\Re ; W)$ of square-summable functions on the group $\mathfrak{R}$ of all integers to the Hilbert space $W$. Obviously the mapping $\Sigma: x(\cdot) \rightarrow \sum_{k=-\infty}^{\infty} U^{k}\{x(k)\}$ is an isometry on $l_{2}(\mathfrak{N} ; W)$ onto $\sum_{-\infty}^{\infty} U^{k}(W)$, i.e. by $(1.4)$ onto $\mathfrak{H}$; and it carries $l_{2}\left(\mathfrak{R}_{0+} ; W\right)$ where $\mathfrak{N}_{0+}=\{k: k \geqq 0\}$ onto $\sum_{0}^{\infty} U^{k}(W)$, i.e. by (1.3) onto $\mathfrak{K}_{0}$. Finally, one verifies easily that

$$
U^{v}\left\{\sum_{k=-\infty}^{\infty} U^{k}\{x(k)\}\right\}=\sum_{k=-\infty}^{\infty} U^{k}\{x(k-v)\}
$$

i.e. the operation $U^{\nu}$ on $\mathfrak{H C}$ corresponds to the translation $\tau_{-\nu}$ on $l_{2}(\mathfrak{R} ; W)$. (q.e.d.)

We shall prove Theorem 1.1 in the continuous case in an analogous spectral-free manner, in contrast to Lax and Phillips who detour into the spectral domain. ${ }^{3}$ In our proof the only hard step is to get the continuous version of the Wold decomposition (1.2). But we already have this in $[7,6.5]$, cf. (4.3) below. ${ }^{4}$ In both prediction and scattering the datum is a unitary group and a subspace invariant under a subsemigroup. The crucial ingredient is thus an isometric semigroup. As shown in [6] the Wold decomposition for such semigroups yields in a natural way the central theorem of prediction as well as the Beurling-Lax Theorem on shift invariant subspaces of the Hardy class $H_{2}$. By starting from this decomposition we thus gain a unified and explicit treatment of the closely related theories of prediction, scattering and shift invariant spaces.

2. Integral reformulation of the discrete Wold decomposition. To get the analogue of (1.2) for isometric flows in which $\mathfrak{R}_{0+}=\{k: k \geqq 0\}$

\footnotetext{
${ }^{3}$ In [5] the translational representation precedes the spectral in the discrete case, but the order is reversed in the continuous case, cf. Chapter II, $\$ \S 1,2$.

4 In [7] we derived (4.3) from the discrete (1.2). Recently Kallianpur and Mandrekar [3] have derived (4.3) from a decomposition given earlier by Cooper [1]. On the other hand, our joint paper [8] cited in $[5$, p. 59] covers only the case corresponding to a 1-dimensional $W$.
} 
is replaced by $[0, \infty)$, we must first recast (1.2) in terms of integrals. ${ }^{5}$ Let

$$
\forall \text { finite } A \subset \mathfrak{N}_{0+}, \quad T(A)=\sum_{k \in A} V^{k} .
$$

Then for any $x(\cdot) \in l_{2}\left(\mathfrak{R}_{0+} ; W\right)$ we have, quite rigorously,

$$
\sum_{k=0}^{\infty} V^{k}\{x(k)\}=\sum_{k=0}^{\infty} T\{k\}\{x(k)\}=\int_{\mathfrak{N}_{0+}} T(d k) x(k) .
$$

In the last integral the operator-valued measure $T(\cdot)$ acts on the $W$-vector-valued integrand $x(\cdot)$, and the result is a vector in 3 . Denote the set of all such vector-integrals for different $x(\cdot)$ in $l_{2}\left(\mathfrak{R}_{0+} ; W\right)$ by $\int_{\mathfrak{R}_{0+}} T(d k)(W) .{ }^{6}$ Then (1.2) becomes

$$
\mathfrak{H}=\mathfrak{H}_{\infty}+\int_{\mathfrak{R}_{0+}} T(d k)(W), \quad \mathfrak{H}_{\infty} \perp \int_{\mathfrak{R}_{0+}} T(d k)(W) .
$$

This is the form which survives in the continuous case after changes are made due to the shift from cardinality to Lebesgue measure. It is convenient to characterize first the operator-valued measure $T(\cdot)$ and its integrals (\$3), and then refer to the harder problem (solved in [7]) of associating such a measure with any isometric flow $\left(S_{t}, t \geqq 0\right)(\S 4)$.

3. Quasi-isometric operator-valued measures and integration. For the measure $T(\cdot)$ defined in $(2.1)$ we see easily that

$$
\begin{aligned}
& \forall \text { finite } A, B \subset \mathfrak{N}_{0+} \& \forall w, w^{\prime} \in W, \quad\left(T(A) w, T(B) w^{\prime}\right) \\
& =\operatorname{card}(A \cap B) \cdot\left(w, w^{\prime}\right) .
\end{aligned}
$$

This suggests the following more general considerations.

Let $W, \mathfrak{X}$ be any (complex) Hilbert spaces, and $\mu$ be any finite, nonnegative, countably additive measure on a prering $\Im$ of subsets of a space $\Lambda$. (We shall denote the not-necessarily-finite Hahn extension of $\mu$ to the $\sigma$-ring $\beta$ generated by $\Im$ by the letter $\mu$ itself.)

s To go from the discrete to the continuous we replace point-functions and their sums by measures and their integrals. We eschew spectral considerations, i.e. involvement with the dual group, for this requires the initial embedding of our semigroup into a group - a step for which we see no need. With this artifice, however, one could derive the continuous version of (1.2) by a spectral detour, either by appeal to the Weyl, von Neumann quantum mechanical relations as in [9] or by the devices used in [5].

S Just as we denote the set of all vector-sums $\sum_{0}^{\infty} V^{k}\{x(k)\}$, for different $x(\cdot)$ in $l_{2}\left(\Re_{0+} ; W\right)$, by $\sum_{0}^{\infty} V^{k}(W)$. 
By a $W$-to-X quasi-isometric measure over $(\Lambda, \Im, \mu)$ we shall mean a function $T$ on $\Im$ such that

(i) $\forall A \in \Im, T(A)$ is a continuous linear operator on $W$ to $\mathfrak{X}$,

(ii) $\forall A, B \in \Im \& \forall w, w^{\prime} \in W,\left(T(A) w, T(B) w^{\prime}\right) \mathfrak{x}$

$$
=\mu(A \cap B)\left(w, w^{\prime}\right)_{w}
$$

Let $T(\cdot)$ be such a measure. ${ }^{7}$ Then precisely as in $[7,5.2]$ we can define $\int_{\Delta} T(d t) x(t)$ for each $x(\cdot) \in L_{2}(\Lambda, ß, \mu ; W)$ so that it is a vector in $\mathfrak{X}$. The set of all such vector-integrals, which we shall denote by $\int_{\Delta} T(d t)(W)$, is a subspace of $\mathfrak{X}$, and we have, cf. $[7,(5.1), 6.2,(6.4)]:^{8}$

3.2. ThEOREM. The mapping: $\Sigma: x(\cdot) \rightarrow \int_{\Delta} T(d t) x(t)$ is a (linear) isometry on $L_{2}(\Lambda, \Theta, \mu ; W)$ onto the subspace $\int_{\Lambda} T(d t)(W)$ of $\mathfrak{X}$.

Now, define $\int_{B} T(d t) x(t)$, where $B \in \mathbb{B}$, to be $\int_{\Delta} T(d t)\left\{\chi_{B}(t) x(t)\right\} .^{\circ}$ Then we see that for $B=\cup_{1}^{\infty} B_{n}, B_{n} \in ß, B_{n} \subseteq B_{n+1}$

$$
\begin{aligned}
& \int_{B} T(d t) x(t)=\lim _{n \rightarrow \infty} \int_{B_{n}} T(d t) x(t), \\
& \int_{B} T(d t)(W)=\text { cls. } \bigcup_{n=1}^{\infty} \int_{B_{n}} T(d t)(W) .
\end{aligned}
$$

Next let $\Lambda$ be an (additive) locally compact abelian semigroup and $\left(S_{t}, t \in \Lambda\right)$ a strongly continuous semigroup of isometries on $\mathfrak{X}$ into $\mathfrak{X}$. Let the prering $\Im$ be invariant under translations $\tau_{t}, t \in \Lambda$, and the operator-valued measure $T(\cdot)$ be "stationary" under $S_{t}$ in the sense that

$$
\forall A \in \Im \& \forall t \in \Lambda, T\left\{\tau_{t}(A)\right\}=T(A+t)=S_{\imath} \circ T(A) .
$$

We then find, cf. $[7,(5.3)(d),(6.3)(d)]$, that

7 (3.1) (ii) entails that $T(A) / \sqrt{ } \mu(A)$ is an isometry on $W$ to $¥$ and that $A \| B$ $\Rightarrow T(A)(W) \perp T(B)(W)$, i.e. $W$ is a "wandering" subspace of the measure $T$. Hence our term "quasi-isometric" and our letter " $W$ ". (3.1) also entails that $T(\cdot)$ is strongly countablyadditive on $\Im$,i.e. $T\left(\cup_{1}^{\infty} A_{k}\right)=\operatorname{sim}_{n \rightarrow \infty} \sum_{1}^{n} T\left(A_{k}\right)$ when $A_{k} \in \Im, A_{k} \| \& \cup_{1}^{\infty} A_{k} \in \Im$. Since $|T(A)|=\sqrt{ } \mu(A)$, we have $T \ll \mu$, but no Radon-Nikodym derivative $d T / d \mu$ exists. Also, the total variation measure $|T|(\cdot)$ of $T(\cdot)$ will in general be $\infty$ on sets $A$ in $\Im$.

8 In [7] we dealt with a specific $T(\cdot)$ over $([a, b], \Im, L e b), a \geq 0$, where $\Im$ is the prering of all bounded open-closed subintervals of $[a, b]$, but our treatment obviously holds for any quasi-isometric measure over any $(\Lambda, \Im, \mu)$.

- The definition makes sense for $B$ in the larger $\sigma$-algebra $Q=\{B: B \subseteq \Lambda$ \& $\forall E \in B$, $E \cap B \in Q\}$; and the resulting $X$-valued measure $\xi_{x}(\cdot)$ is bounded, countably additive and orthogonally scattered on $Q$. Moreover, the measures $\xi_{x}(\cdot), \xi_{y}(\cdot)$ are biorthogonal, cf. $[7,(5.3)]$. 


$$
\begin{aligned}
\forall B \in B \& \forall c \in \Lambda, & S_{c} \int_{B} T(d t) x(t)=\int_{B+c} T(d t) x(t-c), \\
& S_{c} \int_{B} T(d t)(W)=\int_{B+c} T(d t)(W) .
\end{aligned}
$$

This means that the isometry $\Sigma$ of 3.2 carries the operation $\tau_{-c}$ of translation through $-c$ on $L_{2}(\Lambda, \Theta, \mu ; W)$ into the operation $S_{c}$ on $\mathcal{F}$, i.e.

$$
\forall c \in \Lambda, \quad S_{c}=\Sigma \circ \tau_{-c} \circ \Sigma^{-1}
$$

4. Wold decomposition for isometric flows. Let $\left(S_{t}, t \geqq 0\right)$ be a strongly continuous semigroup of isometries on a Hilbert space $\mathfrak{F}_{0}$ to $\mathcal{F}_{0}$, and let

$$
\mathfrak{H}_{\infty}^{0}=\bigcap_{t \geqq 0} S_{t}\left(\mathcal{H}_{0}\right),
$$

$$
\begin{gathered}
W_{0}=\text { the deficiency subspace of }\left(S_{t}, t \geqq 0\right),{ }^{10} \\
T_{0}(a, b]=\text { Rstr. } W_{0}(1 / \sqrt{ } 2)\left\{S_{b}-S_{a}-\int_{a}^{b} S_{t} d t\right\}, \quad(a, b] \in \Im_{0},
\end{gathered}
$$

where $\Im_{0}$ is the prering of all bounded open-closed subintervals $(a, b]$ of $[0, \infty)$. Then, cf. [7, (4.1)-(4.5)],

(4.2) $T_{0}$ is $W_{0}$-to-JC $\mathcal{H}_{0}$ quasi-isometric over $\left([0, \infty), \Im_{0}, L e b.\right)$.

Hence our Theorem 3.2 applies. Furthermore, cf. [7, 6.5],

$$
\mathfrak{F C}_{0}=\mathfrak{F C}_{\infty}^{0}+\int_{0}^{\infty} T_{0}(d t)\left(W_{0}\right), \quad \mathfrak{H C}_{\infty}^{0} \perp \int_{0}^{\infty} T_{0}(d t)\left(W_{0}\right) .
$$

This is the Wold decomposition for the flow $\left(S_{t}, t \geqq 0\right)$, cf. (2.3).

5. Wold decomposition for unitary flows. Now our data is:

(i) $\left(U_{t}, t \in R\right)$ is a strongly continuous group of unitary operators on a (complex) Hilbert space $\mathfrak{H C}$ onto $\mathfrak{H C}$;

(ii) $\mathcal{H}_{0}$ is a subspace of $\mathcal{H}$ such that $U_{t}\left(\mathcal{H}_{0}\right) \subseteq \mathcal{H}_{0}, \quad t \geqq 0$.

On the strength of this data we introduce the following:

5.2. Definition (i) $\mathcal{H}_{\infty}=\bigcap_{t \geqq 0} U_{t}\left(\mathcal{H}_{0}\right)$;

(ii) $W=\mathfrak{H}_{0} \cap\left\{V\left(\mathcal{H}_{0}\right)\right\}^{\perp}$, where $V=I-2 \int_{0}^{\infty} e^{-t} U_{t} d t$;

(iii) $\forall(a, b] \subset R, T(a, b]=$ Rstr. $(1 / \sqrt{ } 2)\left\{U_{b}-U_{a}-\int_{a}^{b} U_{t} d t\right\}$;

(iv) $\forall t \geqq 0 \& \forall c \in R, \quad S_{t}=\operatorname{Rstr} \cdot \mathscr{F}_{0} U_{t}, \quad \mathscr{H}_{c}=U_{c}\left(\mathcal{H}_{0}\right)$.

Then obviously from 5.1 (ii) $\left(S_{t}, t \geqq 0\right)$ is a strongly continuous group of isometries on $\mathfrak{K C}_{0}$ in to $\mathfrak{H C}_{0}$. Hence by (4.3)

${ }^{10}$ I.e. by definition $W_{0}=\mathcal{K}_{0} \cap\left\{V_{0}\left(\mathcal{F C}_{0}\right)\right\}^{\perp}$, where $V_{0}=I-2 \int_{0}^{\infty} e^{-t} S_{t} d t$. 


$$
\mathfrak{F C}_{0}=\mathfrak{F C}_{\infty}^{0}+\int_{0}^{\infty} T_{0}(d t)\left(W_{0}\right), \quad \mathfrak{F C}_{\infty}^{0} \perp \int_{0}^{\infty} T_{0}(d t)\left(W_{0}\right),
$$

where $\mathcal{H}_{\infty}^{0}, W_{0}, T_{0}$ are as in (4.1). It is easy to see that

$$
\mathcal{F C}_{\infty}^{0}=\mathcal{F C}_{\infty}, \quad V_{0}=\operatorname{Rstr}_{\mathcal{F}_{0}} V, \quad W_{0}=W .
$$

Hence from (4.1) and (5.2)(iii)

$$
\forall A \in \Im_{0}, \quad T_{0}(A)=T(A) .
$$

Thus (1) becomes

$$
\mathfrak{H C}_{0}=\mathfrak{H}_{\infty}+\int_{0}^{\infty} T(d t)(W), \quad \mathfrak{K}_{\infty} \perp \int_{0}^{\infty} T(d t)(W) .
$$

To get the analogue of this for $(-\infty, \infty)$, let $\Im$ be the prering of all bounded open-closed intervals $(a, b]$. Then from (5.2)(iii) we see at once that

$$
\forall t \in R \& \forall A \in \Im, \quad T(A+t)=U_{t} \circ T(A) .
$$

Since for any $A, B \in \Im, \exists c \geqq 0$ such that $A+c, B+c \in \Im_{0}$, we infer from (2), (3), (4) and (4.2) that

(5) Tis a $W$-to-अ quasi-isometric measure over ( $($, $\Im, L e b)$.

The results 3.2 and (3.6) therefore apply, and we get:

5.4 THEOREM. The mapping $\Sigma: x(\cdot) \rightarrow \int_{-\infty}^{\infty} T(d t) x(t)$ is an isometry on $L_{2}((-\infty, \infty) ; W)$ onto the subspace $\int_{-\infty}^{\infty} T(d t)(W)$ of FC. Moreover, for all real $c, U_{c}=\Sigma \circ \tau_{-c} \circ \Sigma^{-1}$.

The last equality holds for $c \geqq 0$ as well as for $c \leqq 0$, because by (4) our measure $T$ is stationary under both the semigroups $\left(U_{t}, t \geqq 0\right)$, $\left(U_{t}, t \leqq 0\right)$, and we can therefore apply (3.6) taking $\Lambda=[0, \infty)$ or $(-\infty, 0]$. Likewise we infer from (3.5) that

(6) $\forall$ Borel sets $B \& \forall c \in R$,

$$
U_{c} \int_{B} T(d t)(W)=\int_{B+c} T(d t)(W) .
$$

Now apply $U_{c}, c \in R$, to the subspaces in (5.3). Obviously $U_{c}\left(\mathcal{H}_{\infty}\right)$ $=\mathcal{F}_{\infty}$. Hence from (6), and the fact that $U_{c}$ preserves orthogonality, we get

(7) $\forall c \in R, \quad \mathfrak{K}_{0}=\mathfrak{K}_{\infty}+\int_{0}^{\infty} T(d t)(W), \quad \mathfrak{K}_{\infty} \perp \int_{0}^{\infty} T(d t)(W)$.

From the second assertion in (7) and 3.3(ii) we obtain 


$$
\mathcal{H}_{\infty} \perp \operatorname{cls} . \bigcup_{c \in \mathcal{Q}}^{\infty} T(d t)(W)=\int_{-\infty}^{\infty} T(d t)(W) .
$$

This enables us to conclude from (7) that

$$
\text { cls. } \bigcup_{c \in \mathfrak{R}} \mathfrak{H C}_{c}=\mathfrak{K}_{\infty}+\int_{-\infty}^{\infty} T(d t)(W), \quad \mathfrak{K}_{\infty} \perp \int_{-\infty}^{\infty} T(d t)(W) .
$$

This is the Wold decomposition corresponding to the data (5.1).

6. Deduction of Theorem 1.1. By hypotheses 1.1(ii),(iii), $\mathfrak{H}_{\infty}=\{0\}$ and cls. $\bigcup_{c \in \mathbb{R}} \mathfrak{H C}_{c}=\mathfrak{F C}$. Thus (5.3), (5.5) reduce to

$$
\mathfrak{H}_{0}=\int_{0}^{\infty} T(d t)(W), \quad \mathcal{H}=\int_{-\infty}^{\infty} T(d t)(W) .
$$

Hence by Theorem 5.4, the isometry $\Sigma: x(\cdot) \rightarrow \int_{-\infty}^{\infty} T(d t) x(t)$ is on $L_{2}((-\infty, \infty) ; W)$ onto $\mathcal{H}$, carries $L_{2}([0, \infty) ; W)$ onto $\mathcal{H}_{0}$, and for all real $t, U_{t}=\Sigma \circ \tau_{-t} \circ \Sigma^{-1}$. (q.e.d.)

The accessory Hilbert space $W$ is given by 5.2 (ii), and the isometry $\Sigma$ by 5.4 , i.e. by an integral involving the $W$-to-JC operator-valued measure $T$ of 5.2 (iii).

Having proved Theorem 1.1 in this spectral-free manner, we can deduce the Spectral Representation Theorem $[5, \mathrm{p} .50,3.1]$ by Fourier-Plancherel transformation as in the discrete case $[5, \mathrm{pp}$. $39,40]$. Thus apart from the shift from cardinality to Lebesgue measure we have a common treatment for the discrete and continuous cases.

\section{REFERENCES}

1. J. L. B. Cooper, One parameter semi-groups of isometric operators in Hilbert space, Ann. of Math. (2) 48 (1947), 827-842.

2. P. R. Halmos, Shifts of Hilbert spaces, Crelle's J. 208 (1961), 102-112.

3. G. Kallianpur and V. Mandrekar, Semi-groups of isometries and the representation and multiplicity of weakly stationary stochastic processes, Ark. Mat. 6 (1966), 319335.

4. P. D. Lax and R. S. Phillips, Scattering theory, Bull. Amer. Math. Soc. 70 (1964), 130-142.

5. - Scattering theory, Academic Press, New York, 1967.

6. P. Masani, Shift invariant spaces and prediction theory, Acta Math. 107 (1962), 275-290. 632.

7. —_ Isometric flows on Hilbert space, Bull. Amer. Math. Soc. 68 (1962), 624-

8. P. Masani and J. Robertson, The time-domain analysis of a continuous parameter weakly stationary stochastic process, Pacific J. Math. 12 (1962), 1361-1378.

9. Ja. G. Sinai, Dynamical systems with countable Lebesgue spectrum. I, Izv. Akad. Nauk Armjan SSR Ser. 25 (1961), 899-924.

INDIANA UNIVERSITY 